PAPER

\title{
Electromagnetic function of polymicrogyric cortex in congenital bilateral perisylvian syndrome
}

\author{
R Paetau, J Saraneva, O Salonen, L Valanne, J Ignatius, S Salenius
}

J Neurol Neurosurg Psychiatry 2004;75:717-722. doi: 10.1136/jnnp.2002.004754

See end of article for authors' affiliations ....................

Correspondence to: Dr R Paetau, Epilepsia-pkl, PO Box 280, FIN-00029 HUS, Finland: ritva.paetau@hus.fi

Received 4 October 2002 In revised form 23 November 2002 Accepted

1 November 2003

Background: Congenital bilateral perisylvian syndrome (CBPS) is characterised by bilateral perisylvian polymicrogyria and suprabulbar paresis. Mild tetraparesis, cognitive impairment, and epilepsy are frequently associated. Sensory deficits are surprisingly rare, even though polymicrogyria often extends to auditory and sensorimotor cortex.

Objectives: To study the sensorimotor and auditory cortex function and location in CBPS patients.

Methods: We mapped the sensory and motor cortex function onto brain magnetic resonance images in six CBPS patients and seven control subjects using sources of somatosensory and auditory evoked magnetic fields, and of rhythmic magnetoencephalographic (MEG) activity phase-locked to surface electromyogram (EMG) during voluntary hand muscle contraction.

Results: MEG-EMG coherence in CBPS patients varied from normal (if normal central sulcus anatomy) to absent, and could occur at abnormally low frequency. Coherent MEG activity was generated at the central sulcus or in the polymicrogyric frontoparietal cortex. Somatosensory and auditory evoked responses were preserved and also originated within the polymicrogyric cortex, but the locations of some source components could be grossly shifted.

Conclusion: Plastic changes of sensory and motor cortex location suggest disturbed cortex organisation in CBPS patients. Because the polymicrogyric cortex of CBPS patients may embed normal functions in unexpected locations, functional mapping should be considered before brain surgery.

C ongenital bilateral perisylvian syndrome $(\text { CBPS })^{1}$ is characterised by suprabulbar paresis and bilateral perisylvian polymicrogyria. ${ }^{12}$ Prenatal ischaemia, viral infections, metabolic diseases, ${ }^{3}$ and genetic mechanisms ${ }^{4-6}$ can contribute to polymicrogyria (PMG), which may be four layered, unlayered, or difficult to classify ${ }^{138}$ depending on timing and nature of the underlying cause. Magnetic resonance imaging (MRI) shows PMG over variable areas of the anterior frontal opercular, insular, perisylvian, and sensorimotor cortex. ${ }^{910}$ Bilateral anterior opercular lesions disturb volitional control of the oral and facial muscles, while emotional expressions remain normal. ${ }^{11}$ Patients are typically dysarthric with reduced tongue movements and excessive drooling, and they have increased risk for intellectual disability and epilepsy. ${ }^{16}{ }^{12-14}$

If PMG extends up to the hand and foot sensorimotor cortex, contralateral hemiparesis will ensue. ${ }^{10} 15$ No prominent sensory deficits have been reported even though PMG usually extends to sensorimotor and auditory cortices.

With improved access to MRI, perisylvian PMG is also often found in candidates for surgical treatment of epilepsy. However, little is known about the electromagnetic properties of the PMG cortex or the function and cortical distribution of the sensorimotor cortex in CBPS. ${ }^{16-18}$ Fissural activity in the sensorimotor and auditory cortices is optimally detected by magnetoencephalography (MEG), which allows source localisation with a few millimetres spatial accuracy under optimal conditions and millisecond time resolution. ${ }^{19}$

To study cortex function in CBPS, we analysed the spatiotemporal MEG source patterns of somatosensory and auditory evoked activity, epileptiform activity, and rhythmic motor cortex activity phase-locked to electromyogram (EMG) during hand muscle contraction. ${ }^{20}$

\section{METHODS}

Subjects

Six cooperative CBPS patients (table 1), and seven healthy right handed control subjects gave their informed consent to participate in this study. The study was approved by the ethical committee of the Hospital for Children and Adolescents, University of Helsinki. To optimise signal quality, we included only subjects with normal hearing and a head circumference of $50 \mathrm{~cm}$ or more. Patients P1, P4, and P5 came from a large CBPS family, P3 from another CBPS family, and P2 and P6 had CBPS of unknown aetiology. The control subjects (a sibling of P2, a son of a deceased CBPS patient from the large CBPS family, three students, and two children of the laboratory personnel) had normal head MRIs and lacked signs and symptoms suggestive of perisylvian cortex dysfunction. All patients were dysarthric and had multiple motor disabilities (table 1). In spite of poor fine motor skills, the patients perceived light touch, pain and joint position normally, even in the facial area.

Two neuroradiologists (OS, LV), independently determined the extent of malformation in all MRIs (table 1, figs 2 and 4). Malformed cortex was defined by nodular white-grey border and surface of the cortex as well as abnormal gyration patterns with deep frontoparietal clefts. None of the subjects had heterotopia. The lesions were symmetric in all patients except P6, whose left hemisphere lesion was limited to the frontoparietal operculum sparing the hand area and temporal

Abbreviations: CBPS, congenital bilateral perisylvian syndrome; $E C D$, equivalent current dipoles; EMG, electromyogram; HPI, head position indicator; MEG, magnetoencephalography; MRI, magnetic resonance imaging; PMG, polymicrogyria; PPC, posterior parietal cortex; SEF, somatosensory evoked fields; S/N, signal-to-noise; SI cortex, primary somatosensory cortex; SIl cortex, second somatosensory cortex 
Table 1 Characteristics of the six patients with congenital bilateral perisylvian syndrome

\begin{tabular}{|c|c|c|c|c|c|c|c|c|c|}
\hline & $\begin{array}{l}\text { Age } \\
\text { (years) }\end{array}$ & Sex & $\begin{array}{l}\text { Epilepsy onset- } \\
\text { offset age (years) }\end{array}$ & $\begin{array}{l}\text { Tongue lateral } \\
\text { movements } \\
\text { right/left }\end{array}$ & $\begin{array}{l}\text { Lip movements } \\
\text { right/left }\end{array}$ & $\begin{array}{l}\text { Finger } \\
\text { movements } \\
\text { right/left }\end{array}$ & $\begin{array}{l}\text { Gross motor skills } \\
\text { right/left }\end{array}$ & $\begin{array}{l}\text { Intellectual } \\
\text { capacity }\end{array}$ & PMG location on MRI \\
\hline P1 & 68 & $\mathrm{~F}$ & no & $-1-$ & $w / w$ & $\mathrm{c} / \mathrm{c}$ & $+/+$ & \pm & bi FP, bi Syl \\
\hline P2 & 10 & M & no & $-1-$ & $w / w$ & $\mathrm{c} / \mathrm{c}$ & +/+ & + & bi FP, bi Syl \\
\hline P3 & 12 & $M$ & 14 & $-1-$ & $-1-$ & $\mathrm{c} / \mathrm{c}$ & +/+ & $(-)$ & bi FP, bi Syl \\
\hline P4 & 13 & M & $10,1 \mathrm{sz}$ & $-1-$ & $w / w$ & $c / c$ & +/+ & + & bi FP, bi Syl \\
\hline P5 & 15 & $\mathrm{~F}$ & & $-1+$ & w/+ & $+/+$ & +/+ & \pm & bi Syl \\
\hline P6 & 20 & $\mathrm{~F}$ & $3-12$ & $-1-$ & $+/+$ & $+1-$ & $+/+$ & + & bi Fop, right FP right Syl \\
\hline
\end{tabular}

F, female; $M$, male; +, normal; -, absent; \pm , borderline; (-), mild disability; c, clumsy; w, weak; PMG, polymicrogyria; MRI, magnetic resonance imaging; bi, bilateral; Fop, frontal opercular; FP, frontoparietal; Syl, perisylvian

lobe. Anatomical landmarks of the central sulcus were obvious only in P5 (both hemispheres) and P6 (left hemisphere). The sensorimotor cortex had an abnormal appearance in the remaining nine hemispheres. The temporal plane could be identified in all patients except P3, and it appeared normal only in the left hemisphere of P6.

\section{MEG recordings}

Somatosensory evoked responses were recorded from all subjects, and auditory evoked responses and MEG-EMG coherence were recorded from five patients and five control subjects. One patient and two control subjects were studied before MEG-EMG coherence was included in the protocol. Technical or practical obstacles prevented auditory experiments in two subjects. MEG data were recorded in a magnetically shielded room (Neuromag-122 ${ }^{\mathrm{TM}}$; Neuromag Ltd, Helsinki, Finland) whole-head gradiometer. The subject sat with the head immobile inside the sensor helmet and watched either silent cartoons during somatosensory and auditory stimulation or his or her active hand during the motor task. Before data acquisition, a head coordinate system was created with help of three fiducial points (both preauricular points and the nasion). Three head position indicator (HPI) coils were attached around the scalp and their positions were determined with a three dimensional digitiser (Isotrak; Polhemus Navigation Sciences, Colchester, VT, USA). The reference point was fixed on the scalp to compensate for possible head movement during the digitisation procedure.

Head position relative to the sensor helmet was determined every 5-15 minutes at the beginning of each stimulation block by leading current pulses to the HPI coils. The subjects were monitored continuously through a video camera, and all patients and children were accompanied by a member of the research group in the shielded room. From each subject 60-90 minutes of magnetic brain activity was collected during the experiments, and stored for off-line analysis. Somatosensory and coherence data were sampled at $0.6 \mathrm{kHz}$ and auditory data at $0.3 \mathrm{kHz}$. The recording pass bands were $0.3-190 \mathrm{~Hz}$ and $0.3-90 \mathrm{~Hz}$, respectively.

Somatosensory evoked fields (SEFs) to alternating left and right median nerve electrical stimulation $(0.2-\mathrm{ms}$, constant current pulses triggering motor response) and auditory evoked fields (AEFs) to alternating left and right ear tones (1 kHz, 50-ms sine waves, intensity set individually to comfortable and equally loud perception in each ear) were averaged on-line until satisfactory signal-to-noise $(\mathrm{S} / \mathrm{N})$ ratio was achieved at about 100 averages. $\mathrm{S} / \mathrm{N}$ ratio was judged visually during ongoing stimulation. If no visible responses were discerned, stimulation was continued until 250 averages. Whenever possible, the stimulation procedure was repeated to judge consistency of the responses; thus the final source analysis was based on replicated artefact-free averaged responses to 150-250 stimuli. Large magnetic artefacts $>3000 \mathrm{fT} / \mathrm{cm}$ or eye blinks $>150 \mathrm{uV}$ automatically rejected the concomitant response from the averaging process. A long interstimulus interval of $1.5 \mathrm{~s}$ was chosen not to suppress responses of the second somatosensory cortex (SII) or the auditory evoked magnetic 100-ms response $(\mathrm{Nlm})$ of the youngest subjects.

EMG was recorded from both the first dorsal interosseous muscles (recording pass band $0.3-190 \mathrm{~Hz}$, sampling frequency $0.6 \mathrm{kHz}$ ). The subject was taught to keep the muscle weakly contracted for 4-6 minutes, to produce a rhythmic firing of muscle unit potentials at around $10-40 \mathrm{~Hz}$. To identify the motor cortex, coherence spectra between MEG and rectified EMG signals were averaged over 4-6 minutes of sustained contraction. ${ }^{20}$

\section{Off-line data analysis}

All 122 MEG traces were first screened visually to identify the signal of interest (epileptiform spikes, spontaneous rhythms, evoked responses) and to identify and remove artefacts. Frequency spectra at each sensor were calculated over unfiltered data sets. $\mathrm{Mu}$ rhythm was quantified from amplitude spectra calculated during auditory stimulation and during the motor tasks. Next, the MEG data were displayed as magnetic field patterns and evaluated visually for dipolar fields at each data point over the signal of interest. Finally, dipolar fields were modelled with equivalent current dipoles (ECDs) using a least-squares fit and a spherical MRIguided head model within the Neuromag software. An ECD models the three-dimensional location, orientation, and strength of the current dipole that best explains a measured field pattern and thus represents local cortical activity. If one dipole did not explain all signal components, additional ECDs were found on the basis of differential timing or spatial distribution. Finally, a set of 1-6 dipoles with fixed locations and orientations but freely changing strengths was used to explain the spatiotemporal evolution of all signal components. ECDs were considered adequate if they explained the signal of interest, remained stable in location over a few milliseconds, were physiologically relevant, and if they remained practically unaffected by the other dipoles of a multidipole set.

Sources of MEG activity coherent with EMG signals were determined from cross correlograms (inverse Fourier transform of the normalised cross spectrum at each MEG sensor). The time-domain cross correlogram oscillations typically had dipolar fields, which were modelled with single dipoles fitted to a set of 28 MEG channels around the maximum amplitude oscillation. The largest coherence peak and cross correlogram oscillation were required to occur in the same channels.

Evoked response sources repeatedly found in replicated trials were accepted for further analysis. Spatial accuracy for each response was determined from the distance between replicated sources, which did not differ between the patient and control groups $(7 \mathrm{~mm}$ for the somatosensory $20-\mathrm{ms}$ 
response, $\mathrm{N} 20 \mathrm{~m}, 11 \mathrm{~mm}$ for the somatosensory 35-ms response, $\mathrm{P} 35 \mathrm{~m}$, and $12 \mathrm{~mm}$ for the auditory $\mathrm{Nlm}$ ).

The mean ECD parameters (peak latencies, three dimensional locations, and dipole strengths) of replicated sources were used to represent a subject's response in statistical comparisons (Student's two-tailed $t$-tests).

MEG sources were mapped onto Tl-weighted MRI volumes (Siemens Vision 1.5-T or Magnetom 1.0-T; Siemens, Erlangen, Germany). MRI and MEG data were analysed in the same head coordinate system defined by the three fiducial points (see above).

\section{RESULTS}

\section{Somatosensory responses}

Somatosensory evoked fields (fig l) of the control subjects were in line with previous observations ${ }^{21}$ : the earliest (17$40 \mathrm{~ms}$ ) signals peaked around $20 \mathrm{~ms}$ and $35 \mathrm{~ms}$ after the stimulus and were explained with 1-2 ECDs in the contralateral primary somatosensory (SI) cortex. The contralateral and ipsilateral second somatosensory (SII) cortices in the frontoparietal opercula peaked in $82 \%$ of the trials around $102 \mathrm{~ms}$. The posterior parietal cortex (PPC) in the postcentral sulcus $^{21}$ and the ipsilateral SI in the central sulcus became active around $90 \mathrm{~ms}$ in $36 \%$ of the responses.

In CBPS patients, distorted brain anatomy prevented reliable identification of specific somatosensory areas, and

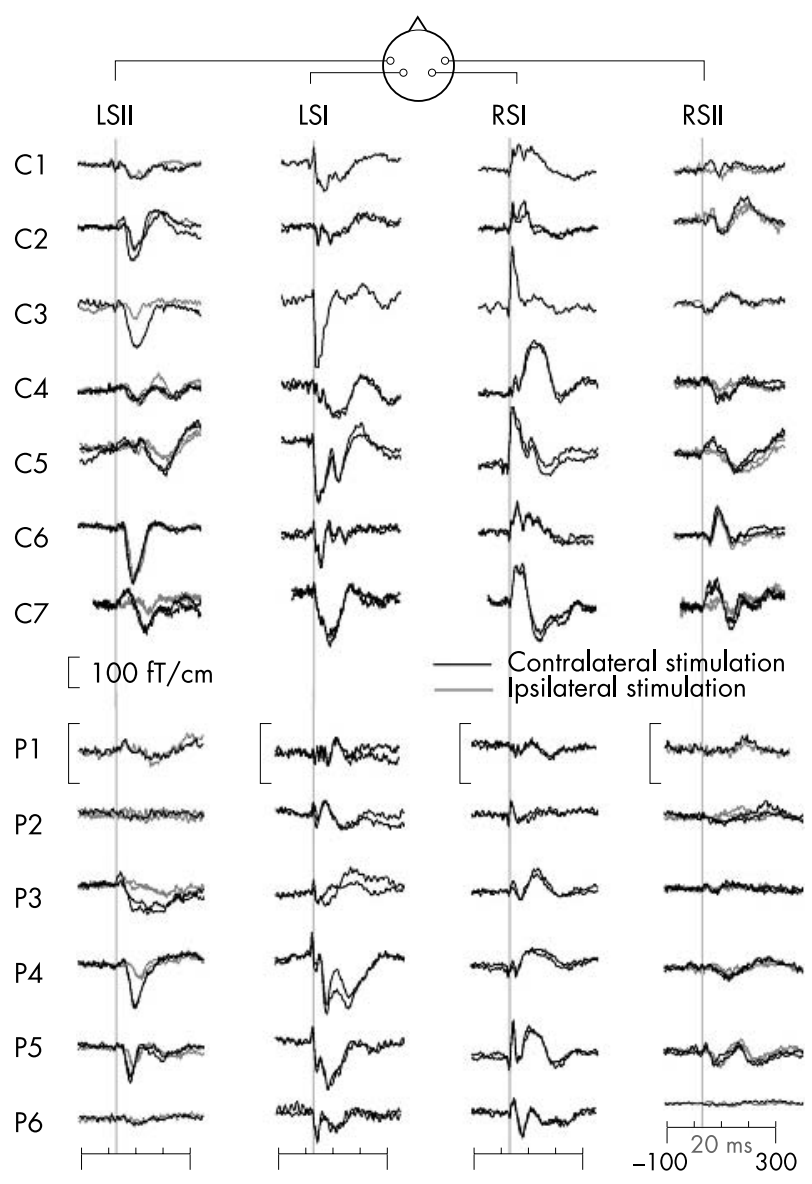

Figure 1 Averaged SEF waveforms of control subjects and patients. Maximum amplitude responses over left and right SI and SIl areas were chosen among 122 planar gradiometer channels. Vertical lines indicate the 20-ms point after stimulus. Responses from repeated trials are superimposed. Note the double scale for responses of P1. Pass band $0.1-90 \mathrm{~Hz}$
SI was defined by sources of the early (17-40 ms) peaks, which occurred at expected latency and slightly reduced amplitude, especially for the right-hemisphere SIc-35 $(p<0.05)$. SI responses originated at the central sulcus in three hemispheres and within the abnormal contralateral frontoparietal cortex in nine hemispheres out of 12 (see figs 2 and 4). Early ipsilateral SI activity occurred in three patients. P2 had left frontal-lobe activity close to midline at $37 \mathrm{~ms}$ and P3 showed ipsilateral parietal 38-ms activity (fig 2, dipole 7). Stimulation of the mildly paretic left hand of P6 elicited simultaneous contralateral and ipsilateral SI activity at $22 \mathrm{~ms}$ and $35 \mathrm{~ms}$. The sources of 20-ms and 35-ms responses were more widely separated in patients than in control subjects (mean \pm SEM: distance $18 \pm 3 \mathrm{~mm}$, range $49 \mathrm{~mm} v 7 \pm 2 \mathrm{~mm}$, range $32 ; \mathrm{p}<0.005)$ Also, more dipoles $(2.3 \pm 0.3$ v $1.5 \pm 0.1$; $\mathrm{p}<0.05$ ) were needed to explain the patients' than the control subjects' SI responses.

All subjects had late 70-90-ms SI/PPC peaks generated at contralateral and/or ipsilateral central sulcus with current orientation perpendicular to the sulcus. Given the localisation inaccuracy of about $1 \mathrm{~cm}$, these late peaks may represent PPC activity as well as true ipsilateral SI responses.

Second somatosensory cortex (SII) activity was defined as any late responses generated lateral and inferior to SI. SII responses occurred less often in patients $(38 \%)$ than control subjects $(82 \%)$. When present, patients' SII source locations varied considerably: some had unusually deep right-hemisphere SII.

\section{Auditory evoked responses}

All auditory evoked responses consisted of a contralateral and ipsilateral 100-ms deflection, $\mathrm{Nl}$, which was the main target of our interest, as well as of later deflections peaking at 180$250 \mathrm{~ms}$. As expected, Nl sources of control subjects were located in the auditory cortex close to the gyri of Heschl in the supratemporal cortex. ${ }^{22}$ A single auditory cortex dipole in each hemisphere explained all auditory responses in three control subjects, while a third source peaked around $125 \mathrm{~ms}$ in two control subjects.

In CBPS patients, $\mathrm{Nl}$ originated in the posterior sylvian fissure on areas appearing polymicrogyric on MRI or immediately adjoining such areas. Additional sources occurred in four patients: unusual activity at 95-190 ms was found in the medial parietal cortex of P1, and P2, P3, and P6 showed one or two additional right or left temporal lobe sources at 100-160 ms. Especially the patients' right hemisphere sources were more numerous, weaker, and a few $\mathrm{mm}$ deeper than those of control subjects (table 2). Systematic latency patterns could neither be seen between ipsilateral and contralateral nor between left and right hemisphere responses.

\section{Spontaneous activity}

Five patients and five control subjects produced centroparietal mu rhythm suppressed during active and passive movements of the contralateral hand. It was exceptionally abundant and only partially suppressed in the right hemisphere of P4. Control subjects had a slightly higher frequency $(9-12 \mathrm{~Hz} v 7-10 \mathrm{~Hz})$ and larger spectral peaks $(22 \pm 5 \mathrm{fT} / \mathrm{cm} v$ $14 \pm 2 \mathrm{fT} / \mathrm{cm}$ ) than patients, but the differences did not reach statistical significance.

Rare epileptiform transients occurred in three patients. Patient 1 had lambda waves in her normal visual cortex, P3 generated sharp waves in the normal-appearing left inferior temporal cortex (and developed psychomotor epilepsy two years later), and P5, with a single rolandic seizure, had bilateral spikes generated by his polymicrogyric frontal opercula. 


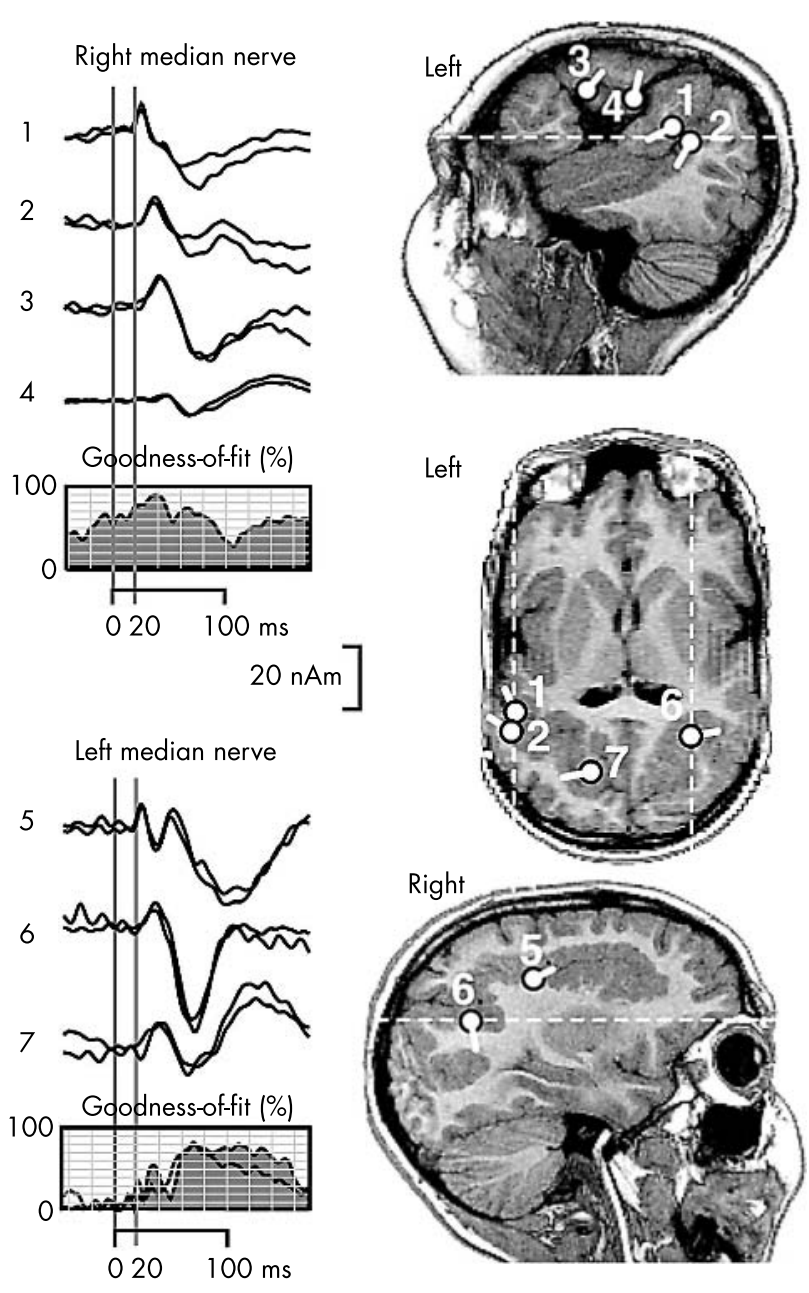

Figure 2 Sources of somatosensory evoked responses of P3. Left: dipole activity as a function of time. Dipoles $1-4$ and 5-7 explain the responses to right and left median nerve stimulation, respectively. Goodness-of-fit shows the percentage of magnetic field variance explained by the set of dipoles. Right: dipole locations (white dots) and orientations (tails) on MRI slices. Dipoles 1 and 2 peak at $23 \mathrm{~ms}$ and $38 \mathrm{~ms}$, respectively, and indicate the left primary somatosensory (SI) cortex. The more anterior dipoles 3 and 4 , peaking at $40 \mathrm{~ms}$ and $90 \mathrm{~ms}$, may reflect activity of the left second somatosensory (SII) cortex. Dipole 5 shows the right SI activity while dipoles 6 and 7 probably explain activity of the contralateral and ipsilateral posterior parietal cortices. Dashed lines indicate section levels.

\section{MEG-EMG coherence and the location of the motor cortex}

EMG of the first dorsal interosseous muscle of all control subjects was coherent with MEG signals recorded over the contralateral hemisphere, although control subjects 2 and 4 showed only weak coherence. Four CBPS hemispheres lacked significant MEG-EMG coherence (fig 3). Maximum coherence amplitudes of control subjects ranged between 0.008 and 0.072 , mean \pm SEM $0.031 \pm 0.007$, and they were not significantly lower in CBPS patients. However, the coherence peaks were wider in control subjects $(11 \pm 1 \mathrm{~Hz})$ than patients $(5 \pm 1 \mathrm{~Hz} ; \mathrm{p}<0.001)$. The main spectral peaks fell in the normal range $(15-35 \mathrm{~Hz})^{20}$ in all control subjects, and in P4 and P5. P2 showed large coherence peaks at an abnormal 8-Hz frequency and $\mathrm{P} 6$ at $38 \mathrm{~Hz}$.

The largest cross correlogram oscillations peaked at $12 \mathrm{~ms}$ (range 22-6 ms) before zero point-that is, MEG signal leading the EMG signal, and had dipolar fields in all control subjects, in P2 and P5 (both hemispheres), and in the left hemisphere of P4 and P6. In control subjects, coherent brain activity was generated close to the motor cortex (MI) in the anterior bank of the central sulcus, within $12 \pm 2 \mathrm{~mm}$ from the earliest ipsilateral SEF sources. In the patients group sources originated within $20 \pm 5 \mathrm{~mm}$ from the early SEF sources and localised to MI in P5 and P6, and to abnormal frontal or frontoparietal cortex in P2 and P4 (fig 4). Thus, normal MEG-EMG coherence was associated with normal central sulcus gross anatomy, and abnormal coherence always occurred with abnormal central sulcus anatomy.

\section{DISCUSSION \\ Study populations}

The clinical symptoms and MRI findings of our patients were similar to those described previously in bilateral perisylvian polymicrogyria, ${ }^{16}{ }^{12-15}$ although our patients had less severe epilepsy and may represent a relatively mild part of the disorder spectrum. We observed no systematic difference between patients with familial and unknown aetiology. The small patient and control groups are, however, likely to preclude detection of some subtle differences and emphasise individual variations.

\section{Origin of MEG signal within PMG cortex}

MEG signals of our patients-especially of the oldest one, $\mathrm{Pl}$-were attenuated compared with control subjects, even though the difference was not always statistically significant.

Extended gyral crowns relative to fissures, could reduce the overall MEG amplitudes, because MEG signals mainly originate in the fissural cortex. Excessive convexial cortex would be expected to increase EEG signals, which are dominated by radial currents. Evoked potentials of some of our patients, and previous studies of evoked somatosensory potentials in other PMG patients ${ }^{16}{ }^{18}$ have shown rather attenuated than enlarged signals, and do not support the hypothesis of relative dominance of radial currents in PMG. Also, the orientation of apical dendrites - that is, orientation of cortical net current may be abnormal in PMG cortex. We found most MEG sources close to big clefts, but the source orientations-for example, dipoles 1, 2, and 5 in fig 2, could be tangential as well as perpendicular to the cortex surface and, in some patients, would agree with currents generated by microgyri.

Nearly normal evoked responses agree with nearly normal glucose metabolism, ${ }^{12}$ proton spectroscopy, ${ }^{23}$ functional MRI, and visual evoked responses ${ }^{24}$ reported in PMG cortex. They also suggest that thalamocortical afferent fibres reach layer IV-III neurones without being stopped by an impenetrable gliotic scar between layers VI and III, as suggested by Golgi analysis for the classic four layered PMG cortex. ${ }^{25}$ Therefore, the four layered PMG may not be valid for our patients, or the macroscopic deformity must embed large cortical areas of normal histology. Little is known about the electromagnetic properties in unlayered or mixed-type PMG, which may be more relevant in familial CBPS. ${ }^{3} 8$

\section{Anomalies of auditory evoked potentials and SEF sources}

PMG areas generated surprisingly normal evoked responses, although some response components originated in unusual locations. The right sylvian fissure-that is, evoked responses of the auditory and of the second somatosensory cortices originated deeper than usual in many patients. Because the corresponding dipoles were relatively weak, such a deep location probably reflects the real anatomy rather than summation of parallel sources over an extended cortical area, mislocalised by the single dipole algorithm. The finding agrees with previous observations, ${ }^{12}$ but its mechanism is unknown. 


\begin{tabular}{|c|c|c|c|c|c|c|}
\hline & \multicolumn{3}{|c|}{ Right hemisphere } & \multicolumn{3}{|c|}{ Left hemisphere } \\
\hline & $\overline{N 1 c}$ & $\mathrm{Nli}$ & $\mathrm{Nlc}+\mathrm{i}$ & $\overline{\mathrm{Nlc}}$ & $\mathrm{Nli}$ & $\mathrm{Nlc}+\mathrm{i}$ \\
\hline $\begin{array}{l}\text { CBPS patients (n) } \\
\text { Latency (ms) } \\
\text { Strength (nAm) } \\
|x|(\mathrm{mm}) \\
y(\mathrm{~mm}) \\
z(\mathrm{~mm}) \\
\text { Control subjects }(\mathrm{n}) \\
\text { Latency: (ms) } \\
\text { Strength: (nAm) } \\
|x|(\mathrm{mm}) \\
y(\mathrm{~mm}) \\
z(\mathrm{~mm})\end{array}$ & $\begin{array}{l}5 \\
101 \pm 4 \dagger \\
24 \pm 5+\dagger \\
46 \pm 3^{*} \\
0 \pm 4 \\
55 \pm 8 \\
5 \\
98 \pm 9 \\
36 \pm 6 \\
55 \pm 1^{*} \\
4 \pm 6 \\
52 \pm 3\end{array}$ & $\begin{array}{l}5 \\
125 \pm 4 \dagger \\
10 \pm 2+\dagger^{* *} \\
50 \pm 4 \\
2 \pm 6 \\
57 \pm 8 \\
5 \\
106 \pm 9 \\
35 \pm 3^{* *} \\
54 \pm 2 \\
3 \pm 5 \\
51 \pm 5\end{array}$ & $\begin{array}{l}10 \\
113 \pm 5 \\
17 \pm 3^{* *} \\
48 \pm 2^{*} \\
1 \pm 3 \\
56 \pm 5 \\
10 \\
102 \pm 6 \\
36 \pm 3^{* *} \\
54 \pm 1^{*} \\
3 \pm 4 \\
52 \pm 3 \dagger\end{array}$ & $\begin{array}{l}5 \\
120 \pm 8 \\
13 \pm 3 \\
45 \pm 3 \\
2 \pm 6 \\
48 \pm 6 \\
5 \\
105 \pm 9 \\
30 \pm 11 \\
53 \pm 3 \\
-3 \pm 2 \\
48 \pm 5\end{array}$ & $\begin{array}{l}5 \\
114 \pm 7 \\
15 \pm 3 \\
49 \pm 4 \\
2 \pm 6 \\
50 \pm 7 \\
5 \\
112 \pm 9 \\
30 \pm 9 \\
50 \pm 4 \\
-2 \pm 6 \\
45 \pm 5\end{array}$ & $\begin{array}{l}10 \\
117 \pm 5 \\
14 \pm 2^{*} \\
47 \pm 2 \\
2 \pm 4 \\
49 \pm 5 \\
10 \\
108 \pm 6 \\
30 \pm 7^{*} \\
51 \pm 2 \\
-3 \pm 3 \\
46 \pm 3 \dagger\end{array}$ \\
\hline \multicolumn{7}{|c|}{$\begin{array}{l}\text { Data expressed as mean } \pm \text { SEM. } \\
|x| \text {, distance from mid-sagittal plane. } \\
x, y \text {, and } z \text {, three dimensional sources coordinates. } \\
{ }^{*} p<0.05,{ }^{* *} p<0.001 \text { for two-tailed } t \text {-test assuming unequal variances: patients } v \text { control subjects. } \\
t p<0.01,+t p<0.001 \text { for paired two-tailed } t \text {-test: contralateral } v \text { ipsilateral or left } v \text { right hemisphere. }\end{array}$} \\
\hline
\end{tabular}

Some CBPS patients had early ipsilateral somatosensory evoked responses. In contrast to normal ipsilateral activity ( $\sim 90 \mathrm{~ms}$ ) mediated by multisynaptic cortico-cortical pathways, the early ipsilateral responses suggest monosynaptic innervation by contralateral thalamocortical axons through the corpus callosum, or by ipsilateral afferent fibres.

Short-latency SI response components could originate in widely separated cortical areas. At least two generators in

\section{A Control subjects}
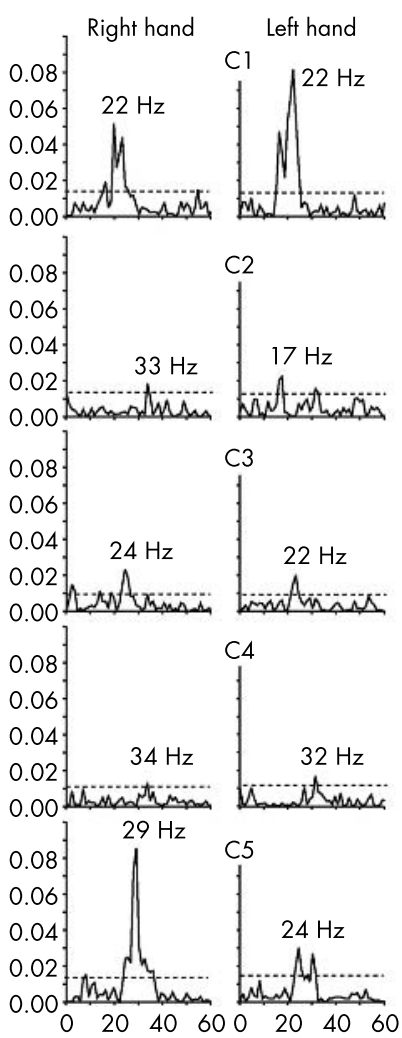

$\mathrm{Hz}$

\section{B CBPS patients}
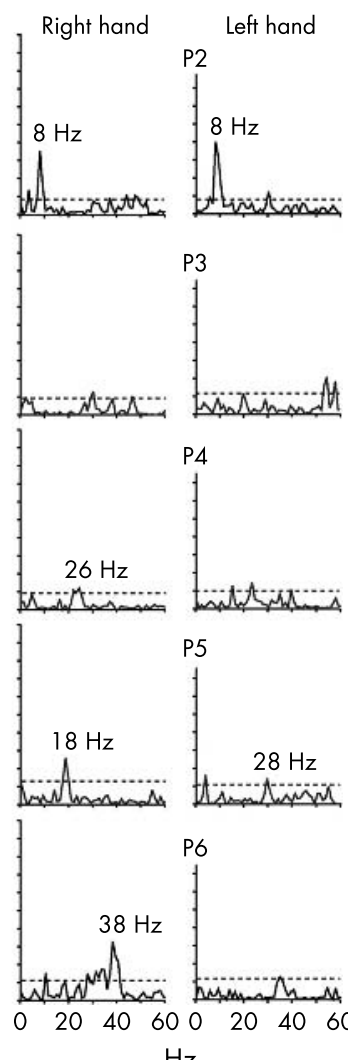

$\mathrm{Hz}$
Figure 3 MEG-EMG coherence spectra of control subjects $\mathrm{Cl}-5$ and CBPS patients P2-6. Maximum left and right hemisphere signals were selected among all 122 gradiometer traces. Dashed horizontal lines indicate the 0.01 significance limit.
Brodmann areas $3 \mathrm{~b}$ and 1 contribute to the early somatosensory evoked responses. ${ }^{26}$ Separation of individual generators by several $\mathrm{cm}$ in some patients agrees best with plastic changes shifting the sites of thalamocortical synapses to the
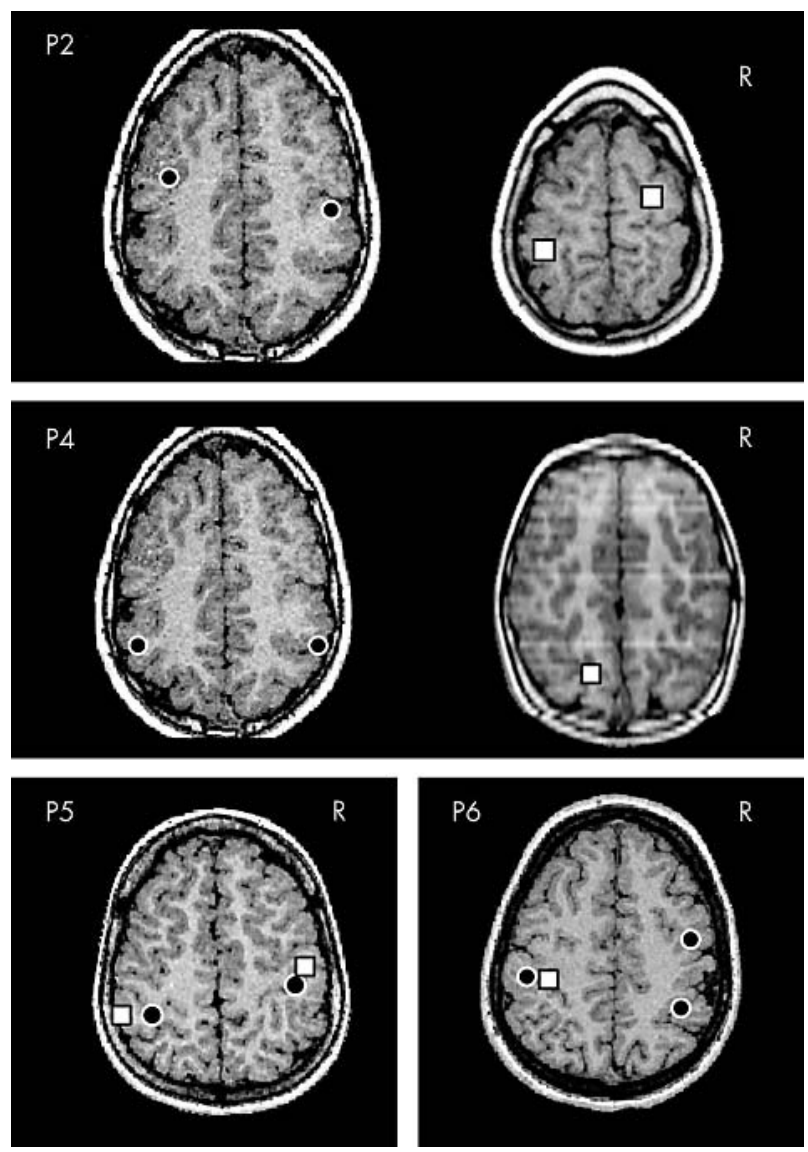

Figure 4 Sources of somatosensory responses to median nerve stimulation at primary somatosensory cortex (circles) and of coherent activities reflecting motor function (squares) mapped on MRI slices. Normal locations are seen in P5 and in the left hemisphere of P6. The circle in the left hemisphere of P6 represents $20-\mathrm{ms}$ and 35 -ms responses to both ipsilateral and contralateral median nerve stimulation; all other circles represent responses to contralateral stimulation. 
periphery of a cortical lesion as shown in experimental four layered $\mathrm{PMG} .{ }^{27}$

\section{Cortex-muscle interaction}

In contrast to robust sensory responses, abnormal cortex did not produce normal oscillations associated with motor function. MEG-EMG coherence was relatively weak in both our control subjects and our CBPS patients. However, in contrast to CBPS patients with weak or absent coherence, all control hemispheres and those three CBPS hemispheres with normal central sulcus anatomy had normal dipolar correlogram fields explained with dipoles in precentral motor cortex. If the central sulcus anatomy was abnormal no MEG-EMG coherence occurred, or it occurred at abnormal frequency, or it had abnormal source locations in the frontoparietal PMG cortex.

The coherence peaks were within normal frequency range except for $\mathrm{P} 2$, whose 8 -Hz coherence indicates an abnormal cortex-muscle interaction. Coherence peaks in the $5-12 \mathrm{~Hz}$ range are occasionally observed in normal subjects, but, in contrast to $\mathrm{P} 2$, these subjects show the strongest coherence in the $15-35 \mathrm{~Hz}$ range. ${ }^{20}$ We thus found a large individual variability both for the strength and frequency of cortical activity coherent with EMG in the CBPS patients. The coherence spectra not only implicate that motor circuitry in many CBPS patients may produce rhythmic activity, resulting in modulation of corticospinal descending commands in the normal $15-35 \mathrm{~Hz}$ range, but also that the rhythmic activity may be abnormally slow or absent reflecting abnormal motor cortical or thalamocortical circuitry in patients with frontoparietal PMG.

\section{Practical considerations}

Individually organised functional circuitries of CBPS patients may reside, at least in part, within PMG cortex. Although our patients did not have severe epilepsy, we hope that our results would encourage careful functional mapping in CBPS patients under evaluation for epilepsy surgery.

\section{ACKNOWLEDGEMENTS}

This work was financially supported by Arvo and Lea Ylppö Foundation, Finska Läkaresällskapet, Helsinki University Central Hospital subsidiaries TLK1117, TLK0278, TRTR019, and T140N09. We thank Drs Seija-Leena Rantala, Auli Nuutila and Eija-Riitta Lauri for patients P2, P3, and P6.

\section{Authors' affiliations}

R Paetau, J Saraneva, S Salenius, Brain Research Unit, Low Temperature Laboratory, Helsinki University of Technology, Espoo, Finland

R Paetau, Hospital for Children and Adolescents, Department of Child Neurology, University of Helsinki, Finland

O Salonen, L Valanne, Department of Radiology, Helsinki University Central Hospital, University of Helsinki, Finland

J Ignatius, Department of Clinical Genetics, Oulu University Hospital, Oulu, Finland

Competing interests: none declared

\section{REFERENCES}

1 Kuzniecky R, Andermann F, Guerrini R. Congenital bilateral perisylvian syndrome: study of 31 patients. Lancet 1993;341:608-12.

2 Becker S. Developmental Foix-Chavany-Marie syndrome: polymicrogyria or macrogyria? Ann Neurol 1990;27:693-4.

3 Barth P. Disorders of neuronal migration. Can J Neurol Sci 1987;14:1-16.

4 Borgatti $\mathbf{R}$, Triulzi F, Zucca C, et al. Bilateral perisylvian polymicrogyria in three generations. Neurology 1999;52:1910-13.

5 Patton MA, Baraitser M, Brett EM. A family with congenital suprabulbar paresis (Worster-Drought syndrome). Clin Genet 1986;29:147-50.

6 Guerreiro MM, Andermann E, Guerrini R, et al. Familial perisylvian polymicrogyria: a new familial syndrome of cortical maldevelopment. Ann Neurol 2000;48:39-48

7 Becker PS, Dixon AM, Troncoso JC. Bilateral opercular polymicrogyria. Ann Neurol 1989:25:90-1.

8 Eriksson SH, Thom H, Heffernam J, et al. Persistent reelin-expressing CajalRetzius cells in polymicrogyria. Brain 2001;124:1350-61.

9 Kuzniecky R, Andermann F, CBPS study group. The congenital bilateral perisylvian syndrome: imaging findings in a multicenter study AJNR Am J Neuroradiol 1994;15:139-44.

10 Barkovich AJ, Kjos BO. Nonlissencephalic cortical dysplasias: correlation of imaging findings with clinical deficits. AJNR Am J Neuroradiol 1992;13:95-103.

11 Weller M. Anterior opercular lesions cause dissociated lower cranial nerve palsies and anarthria but no aphasia: Foix-Chavany-Marie syndrome and "autonomic voluntary dissociation" revisited. J Neurol 1993;240:199-208.

12 Van Bogaert P, David P, Gillain CA, et al. Perisylvian dysgenesis-clinical, $\mathrm{EEG}, \mathrm{MRI}$ and glucose metabolism features in 10 patients. Brain 1998;121:2229-38.

13 Gropman L, Barkovich AJ, Vezina LG, et al. Pediatric congenital bilateral perisylvian syndrome: clinical and MRI features in 12 patients. Neuropediatrics 1997;28:198-203.

14 Guerrini R, Dravet C, Raybaud C, et al. Neurological findings and seizure outcome in children with bilateral opercular macrogyric-like changes detected by MRI. Dev Med Child Neurol 1992;34:694-705.

15 Miller SP, Shevell M, Rosenblatt B, et al. Congenital bilateral perisylvian polymicrogyria presenting as congenital hemiplegia. Neurology 1998:50:1866-9.

16 Scaioli V, Granata T, Battaglia G, et al. The clinical and pathophysiological relevance of evoked potentials study in neuronal migration disorders. In: Spreafico R, Avanzini G, Andermann F, eds. Abnormal Cortical Development and Epilepsy, From Basic to Clinical Science. London: John Libby, 1999:219-26.

17 Yoshiro M, Toshiyuki Y, Kenzo T. Plasticity of central motor and sensory pathways in a case of unilateral extensive cortical dysplasia: investigation of magnetic resonance imaging, transcranial magnetic stimulation, and shortlatency somatosensory evoked potentials. Neurology 1995;45:2255-61.

18 Vossler DG, Wilkus RJ, Pilcher WH, et al. Epilepsy in schizencephaly: abnormal cortical organization studied by somatosensory evoked potentials. Epilepsia 1992;33:487-94.

19 Hari R. Magnetoencephalography as a tool of clinical neurophysiology. In: Niedermeyer E, Lopes da Silva F, eds. Electroencephalography, Basic Principles, Clinical Applications and Related Fields, 3rd ed. Baltimore: Williams \& Wilkins, 1993:1035-61.

20 Salenius S, Portin K, Kajola M, et al. Cortical control of human motoneuron firing during isometric contraction. J Neurophysiol 1997;77:3401-5.

21 Forss N, Hari R, Salmelin R, et al. Activation of the human posterior parietal cortex by median nerve stimulation. Exp Brain Res 1994;99:309-15.

22 Hari R, Aittoniemi K, Järvinen ML, et al. Auditory evoked transient and sustained magnetic fields of the human brain. Localization of neural generators. Exp Brain Res 1980;40:237-40.

23 Kuzniecky R, Hetherington H, Pan J, et al. Proton spectroscopic imaging at 4.1 tesla in patients with malformations of cortical development and epilepsy. Neurology 1997; 48:1018-24

24 Innocenti GM, Maeder P, Knyazeva MG, et al. Functional activation of microgyric visual cortex in a human. Ann Neurol 2001:50:672-6.

25 Williams RS. The cellular pathology of microgyria. A Golgi analysis. Acta Neuropathol 1976;36:269-83.

26 Allison T, McCarthy G, Wood CC, et al. Potentials evoked in human and monkey cortex by stimulation of the median nerve. A review of scalp and intracranial recordings. Brain 1991;114:2465-2503.

27 Jacobs KM, Mogensen M, Warren E, et al. Experimental microgyri disrupt the barrel field pattern in rat somatosensory cortex. Cerebral Cortex 1999;9:733-44. 